\title{
Using agent-based modelling to understand social phenomena
}

Agent-based modelling (ABM) is used to understand the behaviour of a system and
establish what governs its establish what governs its outcomes. Professor Shigeaki Ogibayashi, from the Chiba institute of Technology, the agent-based model is constructed entirely bottom up, it should be possible to build a model where the causal relationship emerging the same as that in the actua system. Together with Kazuya Inc, he applies his innowwar agent-based modelling methodology to reproduce and understand bullying. from the artificial society is

gent-based models are
computer simulations that
attempt to capture the interactions between people, things, places, and time by modelling a system making entities, or agents. Ag decisionmaking the behaviour of a system and establish what governs its outcomes. This individual-based modelling approach can deal with heterogeneity and offers an effective methodology for studying the mechanisms underpinn the dynamic characteristics of social phenomena.

Dr Shigeaki Ogibayashi, Emeritus Professor at the Chiba Institute of Technology, argues that since a set of indispensable behavioural rules causes a specific macrophenomenon, if the model
reproduces the actual phenomenon, then reproduces the actual phenomenon, the
the relationship between the cause and the relationship between the cause
the phenomenon in the model could correspond with the actual cause and the phenomenon in the real world. ABM is therefore the most real-world-friendly

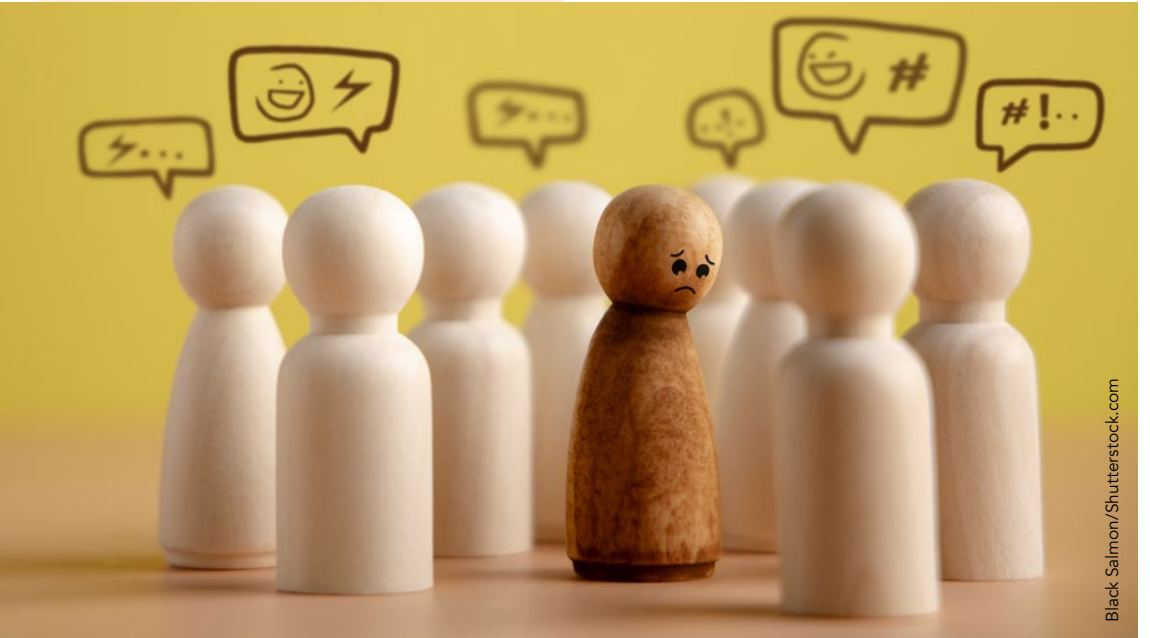

modelling method, making it a promising approach for investigating the causa

MODELUING SOCIAL PHENOMENA Ogibayashi explains that any social or the actions and interations of humans and organisations Moreover, amongs the behavioural rules of humans and organisations there is a particular set of rules pertaining to the cause of the phenomenon in the real social system. Unlike natural phenomena, however. representing the real social world with an equation-based model is actually mpossible due to the complexities of causal relationships in human society. It is also unfeasible to conduct controlled experiments to verify assumed equations, as human behaviour is heterogeneous, and many factors are

INDISPENSABLE FACTORS FOR ABM approach is that if the agent-bapod model is constructed entirely bottomup, then it should be possible to build a model where the causal relationship that emerges from the artificial society is the same as that in the actual system This similarity between the model and the real system can only be realised, however, when the input conditions include a set of indispensable factors for the emergence of the phenomenon of interest. These factors are a set of behavioural rules that can be revealed by a series of computer experiments in which factors are systematically changed one by one. They include the agent types together with their attrib structure. Considering what makes these

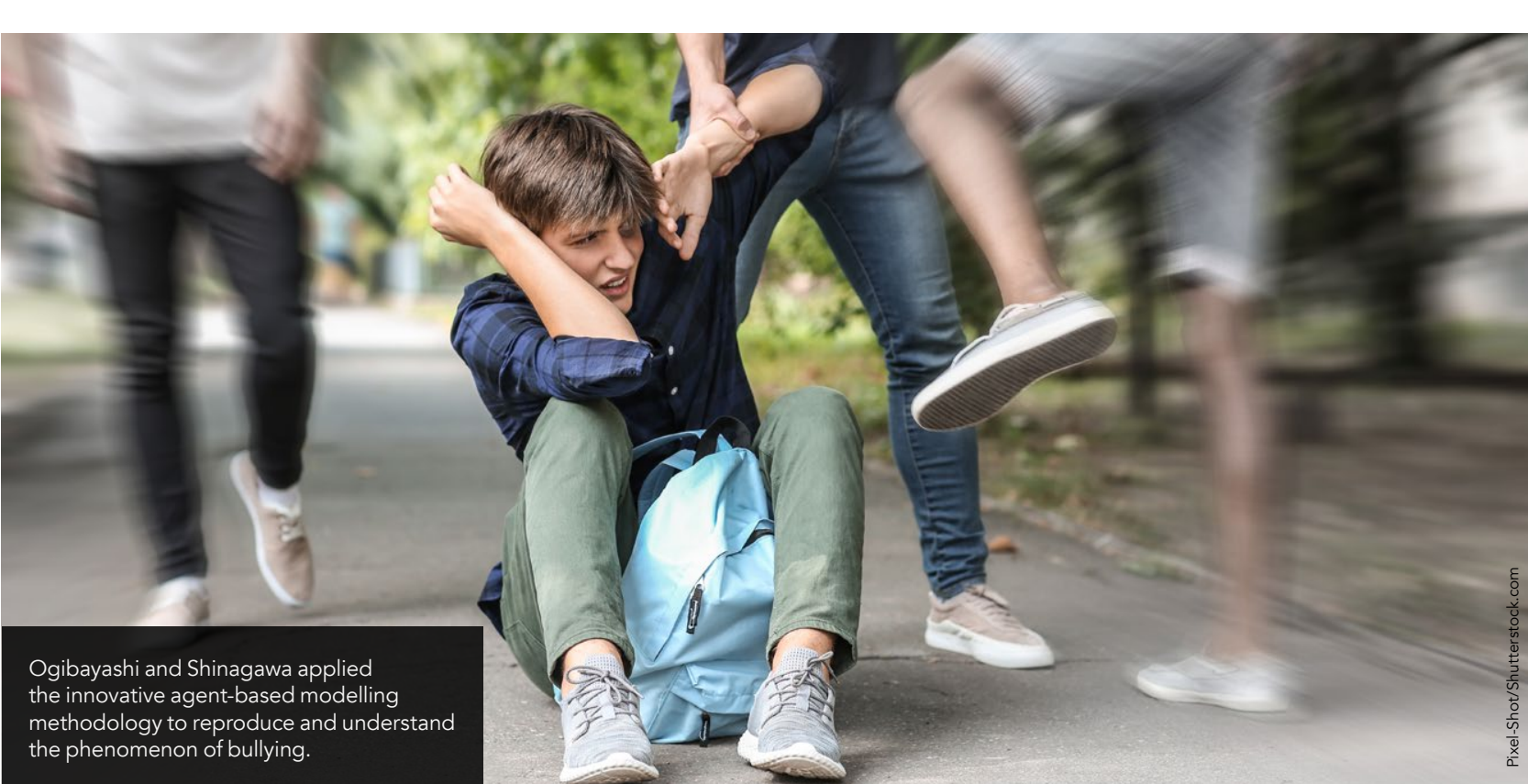

actors indispensable for the emergence of the phenomenon enables a better causal mechanism. Ogibayashi explains that this principle concerning the validity of ABM will enable the set of individually proposed theories previously or currently put forward by social science, to be reconstructed into 'a set of truths on the causal mechanisms of social and economic phenomena'. A proper understanding of these phenomena

in the real world. As a result, ABM has so far received little recognition as a promising methodology for supporting public policies.

Most ABM models proposed in the literature are abstract models - or not entirely bottom-up models - that do not closely imitate real-life processes. Ogibayashi seeks to challenge this, highlighting how ABM can closely would allow

effective policies

to be determined using a group of emerges from the actions and predict their effects
- an approach that anstruction of This makes ABM a promising evpron. for investigating the causal mechanisms of social phenomen

\section{CHALLENGING CURRENT THINKING} Deploying ABM to elucidate the causa mechanism of social and economic phenomena is', says Ogibayashi, 'a new concept in the history of ABM and one which has not yet been widely acknowledged. He observes that many researchers - particularly those in the field of economics - deem ABM insufficiently reliable for the elucidation with tratioch approw with traditional approaches, although it is effective in offering hints on the the heterogeneity of agents as well as the irrationality of decision-making. Moreover, the knowledge required by traditional theories is not necessary in this case, because no aggregaterequired in the case of an entirely botom-up ABM model. ABM, therefore also has promise for forecasting . social problems.

\section{ABM TO UNDERSTAND BULLYING} In a 2020 paper, Ogibayashi and his co-author Kazuya Shinagawa, of aternative approach by using ABM to reproduce and understand the phenomenon of bullying.
Defined in the literature as negative ctions perpetrated by one or more eople toward one or more individual involves unwanted aggressive behaviour and an imbalance of power and strength. The cause of bullying has been the focus of numerous studies, but there is still much to be clarified egarding its underlying mechanism and we are yet to develop fully effective countermeasures.
Many investigations are based on information obtained from interviews with the perspectives of Orisetrats, potential bystanders, and Ogibayashi points out, there is a limit to how well the dynamic characteristics of bullying are explained using

MACROPHENOMENA OF BULLYING . . bullying phenomenon. Ogibayashi and Shinagawa define one of the features of bullying such that five categories of agents emerge through butios, the bulliods and interactions: the thich on the bully's sids - bystanders, agents side of the bullied Anothe fer the bullying is that it involves persistent attack by the perpetrators against the 


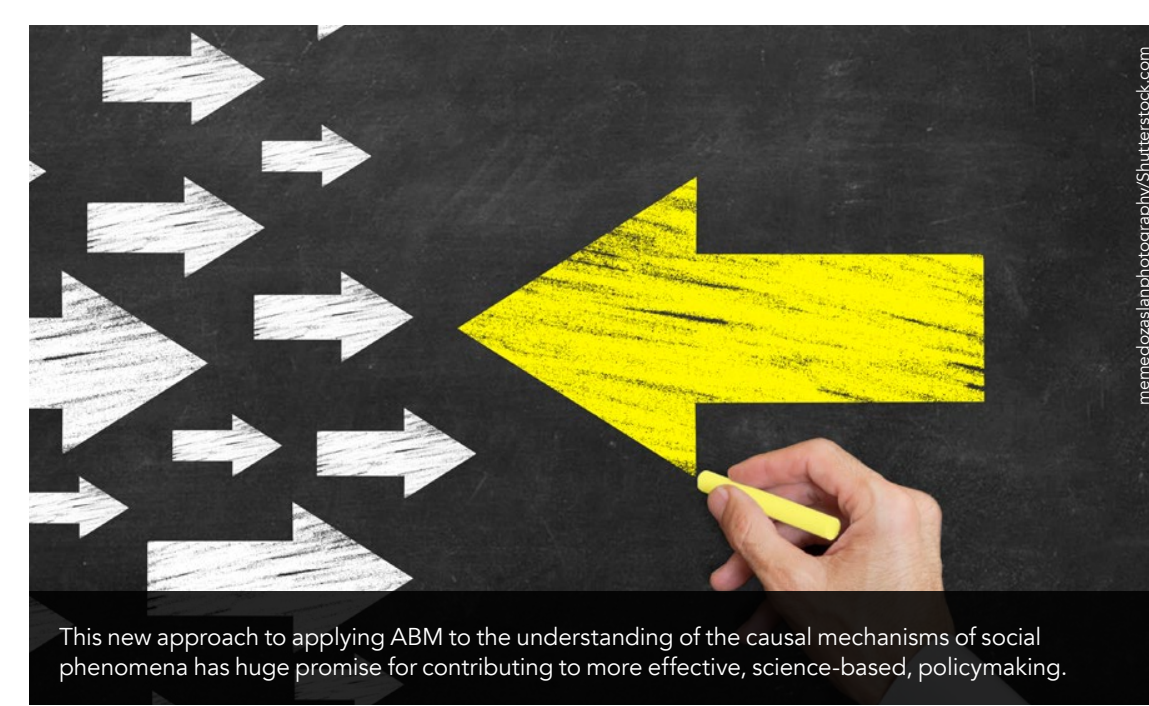

victim which becomes increasingly ESSENTIAL MECHANISMS aggressive as time progresses. The first feature is the most essential in the in the present study, while the emergent mechanism of the second feature remains as a future subject.

In the researchers' model, each agent has a value vector, with each component corresponding to traits in the real world such as skills, preferences, behavioura patterns, etc. The value of each component is initially assigned zero or one at random, but changed to become a shared or non-shared value with other agents due to the interactions among agents. An active agent performed the action, and an objective agent was the object of the action. There were three possible actions. a tuning action, an excluding action, and doing nothing. The tuning action increases the number Agents halues wific thresto for tuning and excluding actions. Pairs, made up of one active agent and one objective agent were selected at random and the active agent performed one of the three actions on the objective agent. This process was then repeated for the remaining agents.

The researchers explain that an agent who frequently excludes others without being excluded themselves often corresponds with the bully or perpetrator. An agent who is more likely to be excluded but doesn't exclude others corresponds with the victim. Other agents who are less likely to be third-party or bystanders. tuning and exclusion characteristics whe are more likely to tup The agents tend to join large groups, and this membership enhances their socia power In contrast those agents who are less likely to tune with others are likely to become solo agents, therefore weakening their social power. The agents' traits and their interactions are reflected in the emergence of the perpetrator and the victim.

INFLUENCING THE MAINSTREAM Ogibayashi's modelling approach has not yet reached the mainstream of $A B M$ research. Unlike traditional ABM- and equation-based approaches, his method facilitates the identification of the indispensable causal factors responsible for a phenomenon. Considering provides a the phenomen. The validity of this innovative ABM model an be assessed First is the agent-specific tendency to
tune with some and exclude others. In this
by howative ABM moll the model reproduces the First is the agent-specific tendency to
tune with some and exclude others. In this by how well the model reproduces the
model, the act of exclusion is assumed to features of the phenomenon. model, the act of exclusion is assumed to

The validlity of this innovative $A B M$ model can be assessed by how well the model reproduces the features of the phenomenon.

be consistent with bullying. Second is that Ogibayashi has applied ABM to other the excluding action is only performed phenomena including the COVID-19 when the perpetrator possesses more pandemic. This model considers both significant power than the agent indispensable These two are the computer experiments rase a series of ther combinations of behavioural rules do not reproduce the phenomenon.

Analysis of the features of each type of agent revealed that the bully is a perso who has a strong tendency to both tun with and exclude others. In contrast, the bullied is a person who is less likely to tune with and exclude others. Furthermore, a typical bystander is more likely to tune with others but less likely to exclude them. This is supported in the literature which identifies the importance of the bystanders' roles in addition to the interaction among the peer group. For the artificial society in the model the process of infection between people
and the process of recovery of each person. Based on medical knowledge of the recovery process, the model considers the number of viruses, virus elimination by immunity, and the enhancement of immunity by antibody production. An indispensable factor for the convergence of the pandemic is that the immunity effect increases with the number of viruses in the body. In the real world, this condition is realised by increased temperature in the human body, which enhances the immunity effect (Ogibayashi, 2022).

This new approach to applying ABM to the understanding of the causal Ogibayashi believes, has humena, Ogibayashi believes, has huge promise science-based, poliormaking

\section{Research Objectives}

Developing the use of agent-based modeling to understand the causes of social phenomen

\section{Detail}

\section{Address}

Address

Bio master's degree in 1971. He worked at the Nippon Steel Corporation from 1971 to 1997 during which period he obtained his PhD at Tokyo University. From 1997 to 2017 he engaged in research and education in social systems science as a professor at Chiba Institute of Technology. He is curenty an emeritus professor of Chiba Institute of Technology and continuing his research focusing on agentbased modeling.

\section{天 $\mathrm{CIT}$}

UU CHIBA INSTITUTE OF TECHNOLOGY
Shigeaki Ogibayashi graduated from the department of physics at Tohoku University, Japan, and obtained his

\section{References}

Ogibayashi, S, (2021) Can Agent-Based Modeling Enable Scientific Policy Making Based on an Understanding of Causa Mechanisms? Journal on Policy and Complex Systems, 7(1), 33-39. doi:10.18278/jpcs.7.1.4

Ogibayashi, S, Shinagawa, K, (2020) Model Structure of Agent-Based Artificial System for Reproducing the Emergence of Bullying Phenomenon. In: Carmichael, T, Yang, Z, (eds) Social Science Society of the Americas. CSS 2018, 229-250. Springer Proceedings in Complexity. Springer.

doi.org/10.1007/978-3-030-35902-7

Ogibayashi, S, (2022) An Agent-Based Model of Infectious Disease that Incorporates the role of Immune cells and Antibodies. In: Yang, Z, Breisen, E, (eds) Proceedings of the 2020 Conference of The Computational Social Science Society of the Americas, CSS 2020. 171-201. Springer Proceedings in Complexity. Springer,
doi.org/10.1007/978-3-030-83418-0

\section{Personal Response}

What further work do you think is needed to encourage the acceptance of $A B M$ as a mainstream

III My further work will be to gather examples of studies on the various social or economic phenomena which
reveal that: a) we can better understand the causal mechanism of each of the phenomena, by revealing the set of indispensable factors to reproduce the phenomenon; and b/ we can assess the effect of various model which includes the indispensable factors relevant
to the phenomenon.
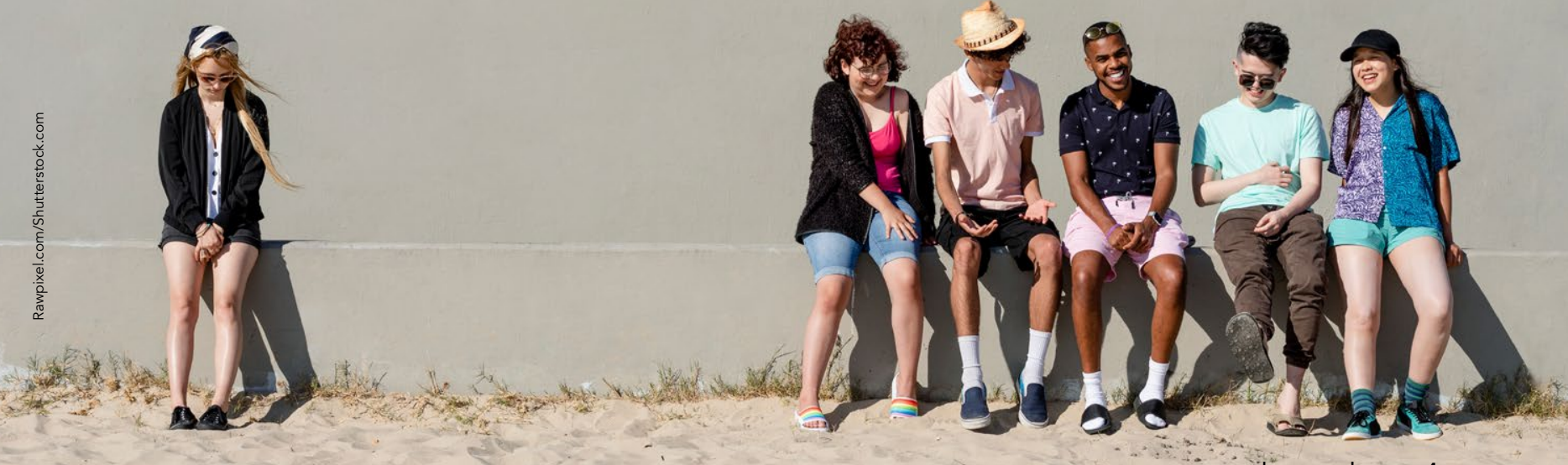